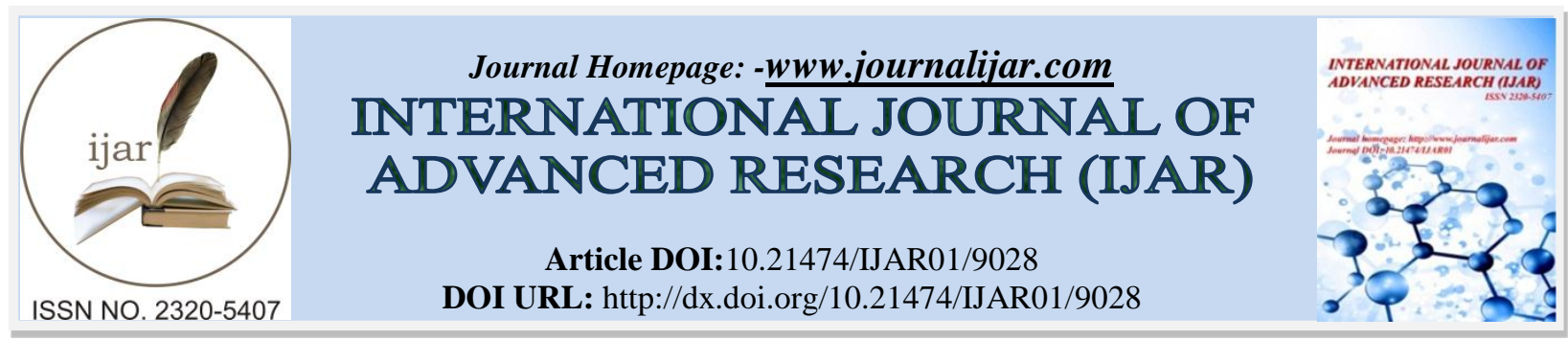

RESEARCH ARTICLE

\title{
GIANT INCISIONAL HERNIA WITH LOSS OF DOMAIN, TREATED SUCCESSFULLY WITH PRIMARY HERNIA REPAIR, SLEEVE GASTRECTOMY, OMENTECTOMY AND SUBTOTAL COLECTOMY.
}

Faiz Tuma ${ }^{1}$, Zhamak Khorgami ${ }^{2}$,Theresa Elder ${ }^{1}$ and John Blebea ${ }^{1}$.

1. Department of Surgery, Central Michigan University College of Medicine, Saginaw, USA.

2. Department of Surgery, The University of Oklahoma College of Medicine, Tulsa, Oklahoma, USA.

\section{Manuscript Info}

Manuscript History

Received: 04 March 2019

Final Accepted: 06 April 2019

Published: May 2019

\begin{abstract}
Giant incisional hernia is a challenging surgical condition, especially in morbidly obese patients, and requires individualized surgical planning and technique. A 54-year-old female with body mass index of $54 \mathrm{~kg} / \mathrm{m}^{2}$ with insulin-dependent diabetes mellitus presented with an extremely large incisional ventral hernia at the site of right subcostal incision from a remote open cholecystectomy. The hernia contained distended stomach, omentum, most of the small bowel, transverse and right colon, and right kidney. After medical optimization, patient underwent sleeve gastrectomy, subtotal colectomy, and primary repair of the hernia. After 10 months, there was no hernia recurrence, the patient's BMI decreased to $37.8 \mathrm{~kg} / \mathrm{m}^{2}$, and she no longer required insulin or oral hypoglycemic medications. In conclusion, concomitant sleeve gastrectomy can be considered with repair of giant incisional hernia in patients with morbid obesity, and colectomy can be considered in cases with loss of domain and long patulous colon.
\end{abstract}

Copy Right, IJAR, 2019,. All rights reserved.

\section{Introduction:-}

Massive abdominal wall hernias have a significant impact on patient quality of life and can pose serious complications such as bowel obstruction or necrosis. We report operative management of an unusual case of a giant abdominal wall incisional hernia in a morbidly obese patient. The hernia contained extremely dilated stomach with intermittent gastric obstruction, small and large intestine, and the entire right kidney and head of pancreas. Treatment of this hernia presented multiple challenges including size and location of the hernia, loss of abdominal domain, and patient's morbid obesity.

\section{Case Presentation}

A 54-year-old female with morbid obesity, hypertension, hyperlipidemia, and diabetes mellitus presented with an extremely large incisional ventral hernia occupying her right upper quadrant. The hernia was chronic for 15 years and it was at the site of a right subcostal cholecystectomy incision. She had been referred for surgical repair of hernia multiple times before presentation, but due to the large size of the hernia, lack of abdominal domain, and her morbid obesity (body mass index of $54 \mathrm{~kg} / \mathrm{m}^{2}$ ), she had been referred to a nutritional team for weight loss. Medical management of weight loss was not successful and she gained an additional $5 \mathrm{~kg}$.

Corresponding Author:-Faiz Tuma.

Address:-Department of Surgery, Central Michigan University College of Medicine, Saginaw, USA. 
At the time of presentation, she had a very large hernia $(30 \times 40 \times 25 \mathrm{~cm})$ in the right upper quadrant, irreducible, with nausea and vomiting. Her lab results indicated volume depletion with acute kidney injury (WBC $27.2 \mathrm{x} 10^{9} / \mathrm{L}$, Lactate $5.2 \mathrm{mmol} / \mathrm{L}$, and Creatinine $2.4 \mathrm{mg} / \mathrm{dL}$ ). After initial resuscitation, abdominal $\mathrm{x}$-ray showed a large hernia containing the stomach with nasogastric tube and loops of intestine (Fig. 1). CT scan revealed a massive hernia containing extremely distended stomach, small and large intestine, omentum, and head of pancreas, along with significant proximal gastric dilation (due to distal obstruction), and significant attenuation of mesenteric vessels and angulation of aorta (Figure 2). She also had pneumonitis, possibly due to aspiration. Gastric decompression was performed. Upper gastrointestinal (GI) series showed no contrast beyond the duodenal bulb. An esophagogastroduodenoscopy was performed which showed distended stomach with distal obstruction and acutely inflamed gastric antrum due to distortion from the patient's large hernia.

Surgical repair was performed after medical optimization. The patient underwent open surgery with wide a right subcostal skin incision. Hernial contents were explored (Fig. 3). To allow reduction of abdominal contents, sleeve gastrectomy, omentectomy, and subtotal colectomy with primary ileosigmoid anastomosis was performed which increased the remaining size of her abdominal cavity. After replacement of the remaining abdominal contents, excision of redundant abdominal wall and skin and primary repair of the $20 \mathrm{~cm}$ right upper quadrant hernia defect was performed (Fig. 4).

Epidural analgesia was used for perioperative pain control. The patient had an uneventful postoperative course with no postoperative complications. Postoperative upper GI contrast study did not show any leak or outlet obstruction. She was discharged home on postoperative day 10. At 10 month follow-up, there was no recurrence of hernia. She has lost $36 \mathrm{~kg}$ (body mass index of $37.8 \mathrm{~kg} / \mathrm{m}^{2}$ ), is off insulin and oral hypoglycemic medications, and is able to ambulate without preoperative walking aids.

\section{Discussion:-}

Up to $8 \%$ of morbidly obese patients may have a ventral hernia (Datta et al. 2008). Obesity-related changes in abdominal wall and increased abdominal wall pressure may predispose this group to hernias. Incisional hernias occur in up to $20 \%$ of abdominal surgeries (Eriksson et al., 2014) with higher frequency in obese patients (Sugerman et al., 1996).

We presented a morbidly obese patient with extremely large incisional hernia at the site of right subcostal incision for a remote cholecystectomy. Most of her bowel was contained within the hernia sac and retroperitoneal structures and vasculature were displaced. After years of discomfort, her acute presentation was for gastric obstruction and severe distention of stomach. The patient suffered obesity-related comorbidities, including diabetes mellitus requiring high doses of insulin. The primary challenge in surgical treatment of this patient was loss of abdominal domain with most intraabdominal viscera contained within the hernia sac for years. Choice of closure technique of the hernia defect (primary versus mesh) was another challenge in surgical planning. The patient's colon was elongated and incarcerated within the hernia sac and resection was part of the preoperative planning. The sleeve gastrectomy of an extremely dilated stomach not only helped with reduction of intraabdominal contents but also helped to manage her obesity and diabetes mellitus very effectively in 10 months follow-up. Reduction of retroperitoneal structures was performed with minimal mobilization. The fascial defect was closed primary with interrupted 0-Vicryl sutures. Postoperative care included respiratory support on postoperative day 1 and aggressive pulmonary toilet after extubation, upper GI series to rule out leak, strict glycemic control, and physical therapy support of ambulation.

Repair of giant abdominal wall hernias presents a challenge. Component separation is a well-established technique for repair of complex hernias (Pauli et al., 2013); but it was not applicable to this case with a giant subcostal defect with extension to the lateral part of abdomen. Bridging repair using mesh is another option for large hernias (Bikhchandani et al., 2013). We chose not to use mesh in this case after her colectomy and stomach resection allowed closure of the abdomen without tension.

A systematic review of giant incisional hernia repair by Datta et al. (2008) showed that this procedure has a high morbidity (median 32\%), however most evidence about the subtype of hernia repair is retrospective with a limited number of patients (Eriksson et al., 2014). Therefore, selection of surgical technique should be individualized based on patient characteristics, hernia site, and contents of the hernia. Hernia recurrence is a major concern with a rate between $10 \%$ and $30 \%$ (Holihan et al., 2016). Performing a bariatric procedure in a morbidly obese patient with 
abdominal wall hernia may help with resolution of morbid obesity and obesity-related comorbidities, and lower the risk of hernia recurrence after hernia repair. Ventral hernia repair in obese patients (BMI $>30 \mathrm{~kg} / \mathrm{m}^{2}$ ) has been reported with 20-27\% recurrence rate (Froylich et al., 2016). Bariatric surgery and ventral hernia repair in morbidly obese patients can be performed in two stages (with bariatric procedure as the first and hernia repair later after weight loss), or concomitant when hernia repair is urgent. The staged approach may be more preferred since adding ventral hernia repair to an elective bariatric surgery can significantly increase the rate of postoperative complications (Khorgami et al., 2015).

\section{Conclusion:-}

Repair of huge incisional hernia can be challenging, especially in morbidly obese patients. Concomitant bariatric surgery (e.g. sleeve gastrectomy) may be helpful in this group of patients by inducing postoperative weight loss, and decreasing the risk of hernia recurrence. Colectomy can be considered in cases with loss of domain and redundant colon.

\section{Conflicts of Interest:}

The authors declare that there are no conflicts of interest regarding the publication of this paper

\section{Funding}

This research did not receive any specific grant from funding agencies in the public, commercial, or not-for-profit sectors.

Figure 1:-Abdominal x-ray shows most of colon and viscera outside of abdominal cavity

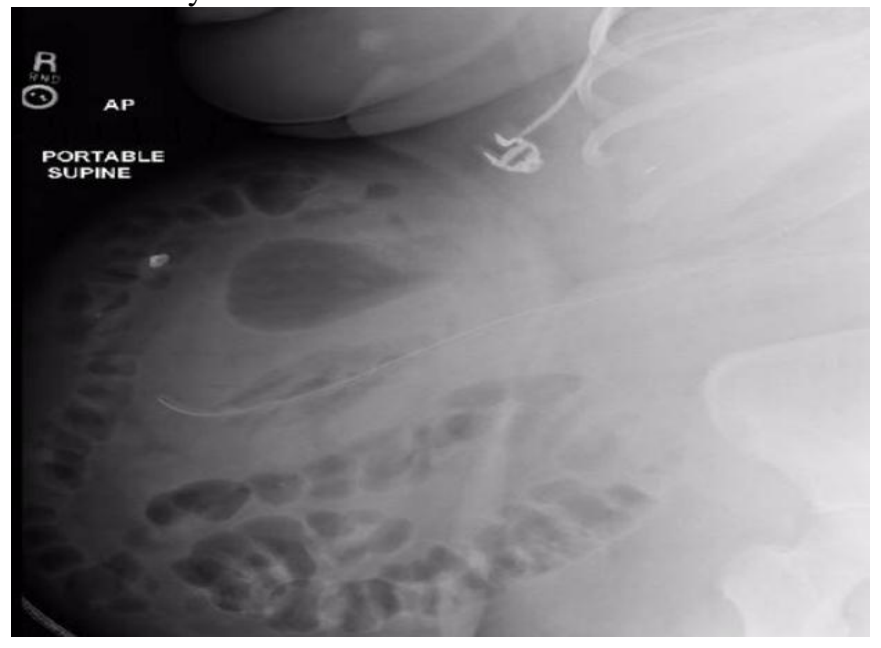

Figure 2:-Abdominal CT scan shows massive gastric distention with extension into the right lateral abdominal wall hernia (A). There is marked displacement of vascular structures (aorta and its main branches) to the right (B).

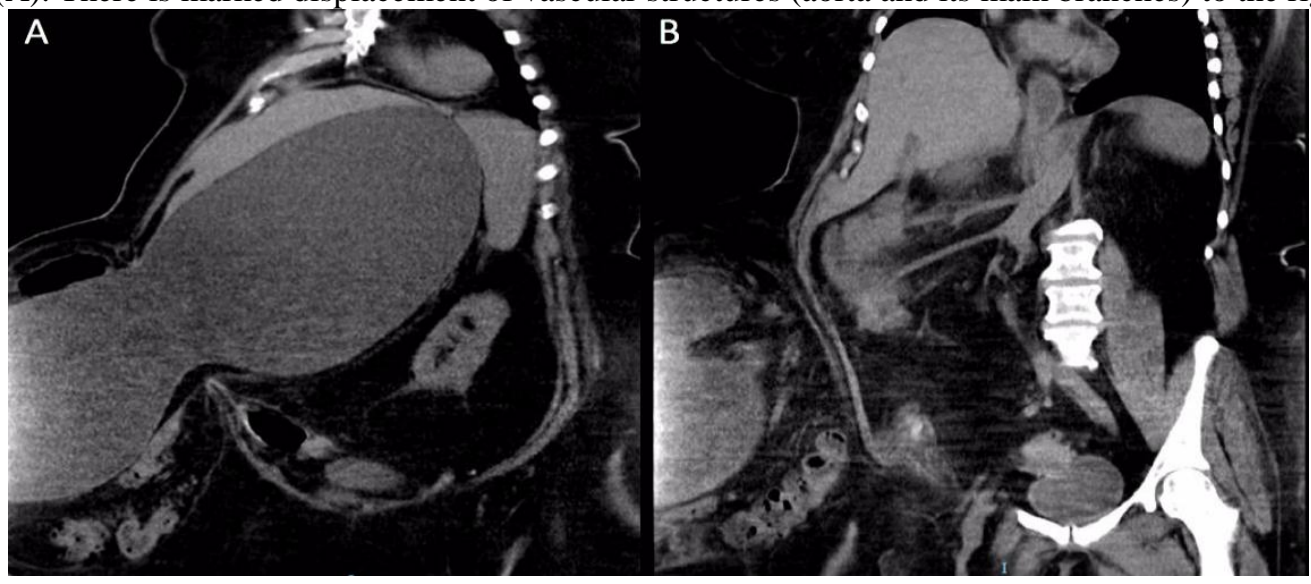


Figure 3:-Giant hernia content explored.

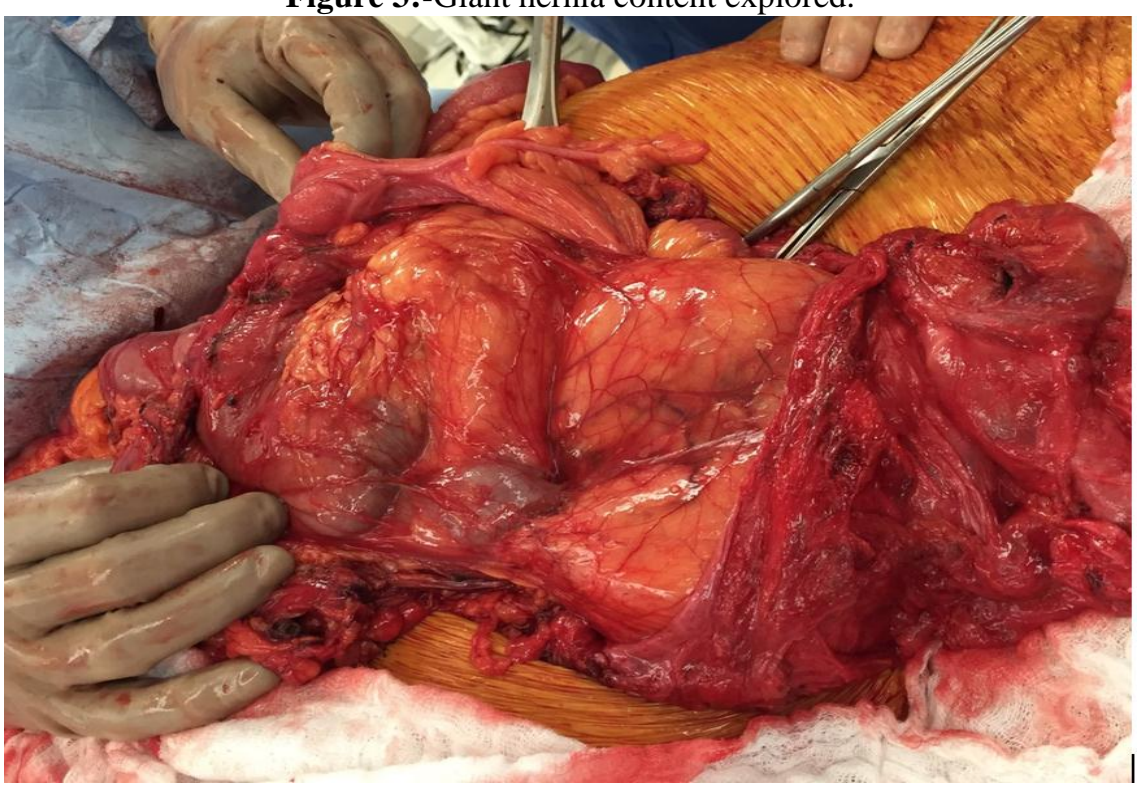

Figure 4:-Reduction of hernia content and primary closure of the defect.

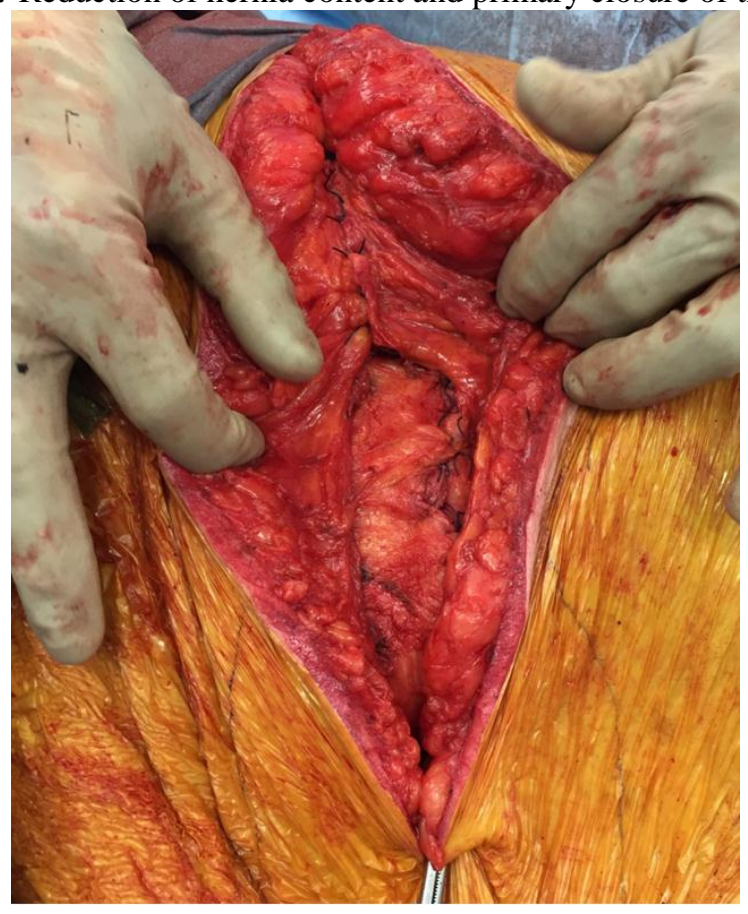

\section{References:-}

1. Datta T, Eid G, Nahmias N, et al. Management of ventral hernias during laparoscopic gastric bypass. Surgery for obesity and related diseases : official journal of the American Society for Bariatric Surgery. Nov-Dec 2008;4(6):754-757.

2. Eriksson A, Rosenberg J, Bisgaard T. Surgical treatment for giant incisional hernia: a qualitative systematic review. Hernia : the journal of hernias and abdominal wall surgery. Feb 2014;18(1):31-38.

3. Sugerman HJ, Kellum JM, Jr., Reines HD, et al. Greater risk of incisional hernia with morbidly obese than steroid-dependent patients and low recurrence with prefascial polypropylene mesh. American journal of surgery. Jan 1996;171(1):80-84. 
4. Pauli EM, Rosen MJ. Open ventral hernia repair with component separation. The Surgical clinics of North America. Oct 2013;93(5):1111-1133.

5. Holihan JL, Askenasy EP, Greenberg JA, et al. Component Separation vs. Bridged Repair for Large Ventral Hernias: A Multi-Institutional Risk-Adjusted Comparison, Systematic Review, and Meta-Analysis. Surgical infections. Feb 2016;17(1):17-26.

6. Bikhchandani J, Fitzgibbons RJ, Jr. Repair of giant ventral hernias. Advances in surgery. 2013;47:1-27.

7. Froylich D, Segal M, Weinstein A, et al. Laparoscopic versus open ventral hernia repair in obese patients: a long-term follow-up. Surgical endoscopy. Feb 2016;30(2):670-675.

8. Khorgami Z, Andalib A, Corcelles R, et al. Concurrent Ventral Hernia Repair in Patients Undergoing Laparoscopic Gastric Bypass and Sleeve Gastrectomy: A Case-Matched Study Utilizing the National Surgical Quality Improvement Program (Nsqip) Database. Surgery for Obesity and Related Diseases. 2015;11(6):S33. 